\title{
DIGITAL SCINTIGRAPHY: CONCEPTS AND DESIGNS
}

\author{
Sebastian Genna, Sing-Chin Pang, and Andrew Smith \\ Veterans Administration Medical Center \\ Boston, MA. 02130 \\ Boston University Medical Center \\ Boston, MA. 02118
}

\section{ABSTRACT}

Fundamentals of a 1-D digital camera are reviewed in order to introduce new designs and concepts having broader applications. A digital iterative position analysis procedure accounts for coordinate response interdependencies in a 2-D camera which would otherwise result in image nonlinearities and nonuniformities. Faster position analysis is made possible by a circuit employing buffered analog pulse current integrators which are multiplexed with an analog-to-digital converter. These digital camera designs, along with a digital method of rejecting pulse pileup which is currently under development, are incorporated in the design of a fast high sensitivity stationary single crystal scintillation camera. The design employs fixed $z$ collimation and rotatable slit $\theta$ collimation to collect data for single photon computerized tomography applications.

\section{INTRODUCTION}

In a digital camera the position of a scintillation event is computed from digitally-converted photomultiplier pulse charges. Such a procedure makes it possible to more fully utilize the position and statistical information contained in photomultiplier responses to

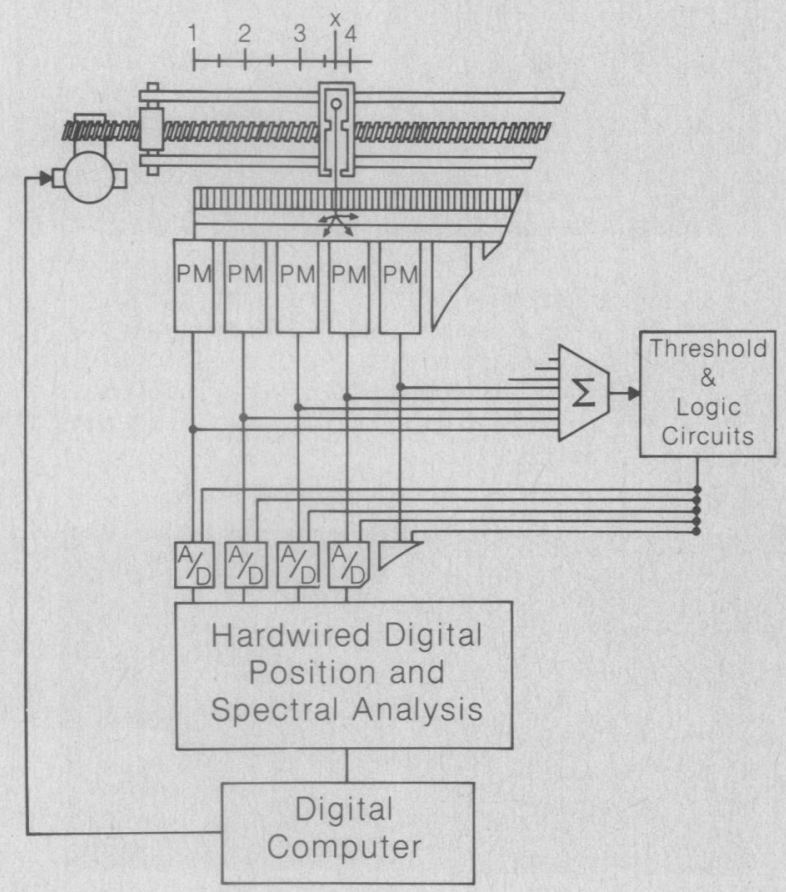

Fig. 1 Schematic of 1-D detector and source positioning apparatus. Computer positioned narrow gamma-ray beam ( $1 \mathrm{~mm}$ diameter) is used to initialize and calibrate camera. Threshold and analog logics select pulses and trigger pulse integration and digital conversions. Calibration is by digital computer while digital position and spectral analysis are hardwired. scintillation events $(1,2)$ and thus permit small improvements in energy and position resolution to be obtained. More significantly, a digital analytical procedure is capable of producing superior event-rate and uniformity characteristics and is particularly applicable to more complex gamma-camera geometries. Some fundamentals of one-dimensional position analysis, which are based upon digital camera developments elaborated on elsewhere (1), are outlined in the first section of this paper in order to facilitate the discussion of new concepts and designs. These consist of: (1) a two-dimensional iterative positon analysis method which accounts for coordinate response interdependencies, (2) a fast pulse integration and digital conversion method, (3) digital pulse pileup rejection methods, and (4) a design for a high event-rate singlephoton emission computerized tomography camera which incorporates these methods.

\section{ONE-DIMENSIONAL CAMERA}

Figure 1 illustrates schematically a one-dimensional camera and a source positioning apparatus used to calibrate it. In response to a scintillation event, $i$, the photomultipliers generate a pulse charge, $Q_{i}{ }^{J}$, where the superscript, J, identifies the photomultiplier. Each $Q_{i}{ }^{J}$ is sampled and digitally-converted. A subsequent division by the sum of these digital signals from all the photomultipliers yields a normalized photomultiplier response, $q_{i}{ }^{\mathrm{J}}$, which is proportional (within statistics) to the fraction of the collected scintillations which is received by the $\mathrm{J}^{\text {th }}$ photomultiplier from the $i^{\text {th }}$ event. These are the input data for digital position analysis.

Figure 2 illustrates the normalized response characteristics of each of the seven photomultipliers in the one-dimensional camera (Fig. 1). Each point on the curve of the $\mathrm{J}^{\text {th }}$ photomultiplier is the average

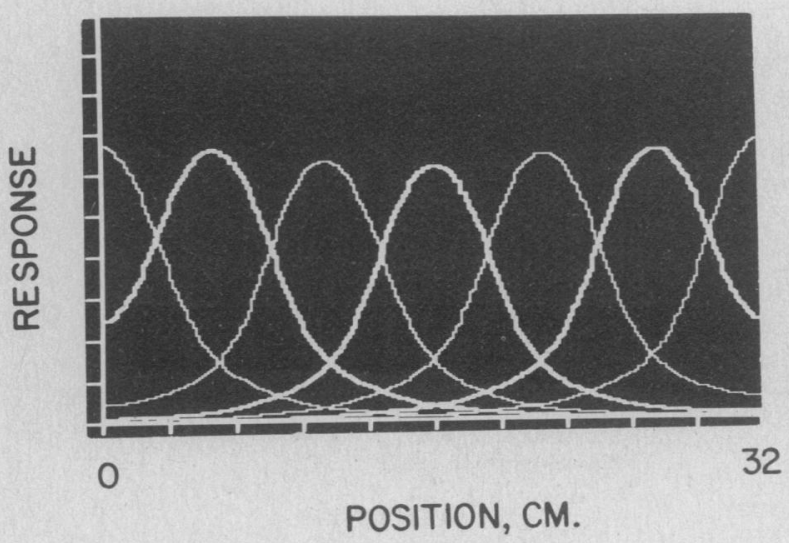

Fig. 2 Normalized photomultiplier response characteristics. Ordinate is average normalized response (fractional pulse charge averaged over 400 events/location) from each of seven photomultipliers as function of position, $x$, of calibrating source. 
normalized response, $\mathrm{q}^{\mathrm{J}}$, obtained from a statistically adequate number of events at the location $\mathrm{x}$ (produced by a $1 \mathrm{~mm}$ narrow-beamed gamma-ray source). Thus, within the limits of statistical uncertainties and the spatial double-valuedness of the bell-shaped characteristic curves, each photomultiplier can provide a separate determination of a position, $x_{i}{ }^{J}$, for each event. As will be shown later, removal of the double-valuedness, which is necessary for position evaluation, leaves high residual uncertainties near tube centers.

By statistical analysis of the characteristic curves (Fig. 2), one can assign a reliability, $w_{i}{ }^{J}$, to each position, $x_{i}{ }^{J}$, determined as a function of a photomultiplier response function, $q_{i} \mathrm{~J}$. Figure 3 illustrates such a determination, as a function of $x$, which was derived for the central tube of the one-dimensional array. At the central region of the photomultiplier the reliability is set to zero, primarily as a result of large double-valuedness uncertainties.



POSITION, CM.

Fig. 3 Reliability weighting function from central photomultiplier as function of source position. Reliability drop in central tube region due to doublevalue uncertainty.

Figure 4 illustrates the position analysis digital circuitry, functionally. Digital $Q_{j}{ }^{J}$ are normalized to $q_{i}{ }^{J}$. Adjacent $q_{i}{ }^{J-1}$ and $q_{i}{ }^{J+1}$ are compared to determine whether the event occurred to the left (L) or right (R) of the $\mathrm{J}^{\text {th }}$ tube center in order to remove double-valuedness. The reference tables are addressed and values of $(w x)_{i}{ }^{J}$ and $w_{i}{ }^{J}$ are extracted as a function of $\mathrm{q}_{i}{ }^{\mathrm{J}}$ for each event. The function

$$
\left\langle x_{i}\right\rangle=\frac{\Sigma_{J} w_{i}^{J}}{\Sigma_{J} w_{i}^{J}}
$$

is then evaluated and stored in memory provided that the $i^{\text {th }}$ event falls within a prescribed energy window, as a function of $x$. A similar window-analysis may be provided for the summation of the photomultiplier reliability weighting functions, $\Sigma_{\mathrm{JW}} \mathrm{w}(\mathrm{x})$, for pulse pileup rejection reasons as discussed in the next section.

\section{TWO-DIMENSIONAL CAMERA}

A scintillation camera may be described in terms of its event-rate and position analysis characteristics. Event-rate may be described by the speed, or data-rate, at which image pixels can be accumulated and the degree to which pulse pileup can be eliminated or accounted for. A problem peculiar to two-dimensional scintigraphy (as compared to a one-dimensional camera) is that a camera cannot truly respond to a source's coordinates, $\mathrm{x}$ and $\mathrm{y}$, independently.

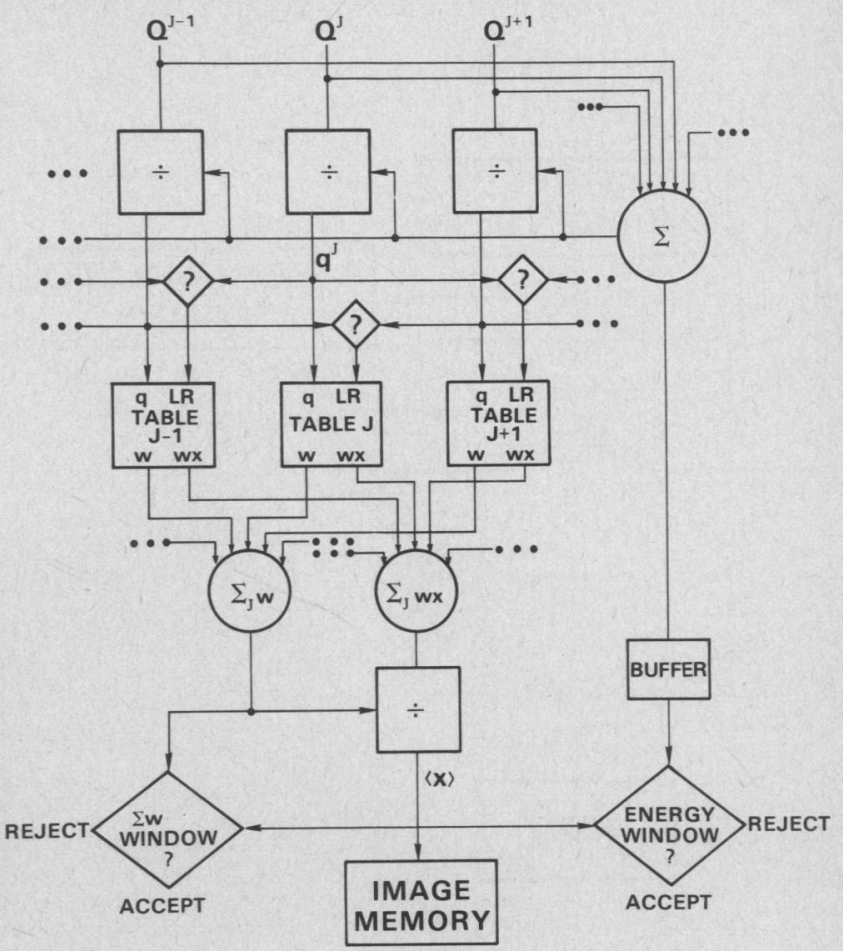

Fig. 4 One-dimensional digital position analysis circuitry. The normalized pulse charge, $\mathrm{q}^{\mathrm{J}}$, is evaluated for double-valuedness by comparing adjacent signals, $q^{\mathrm{J}-1}$ and $\mathrm{q}^{\mathrm{J}+1}$, causing left (L) or right (R) table to be addressed with $\mathrm{q}^{\mathrm{J}}$. A position $\langle\mathrm{x}\rangle$ is determined from extracted $\mathrm{w}^{\mathrm{J}}$ and $(\mathrm{wx})^{\mathrm{J}}$ and stored if $\Sigma Q^{J}$ passes through energy window (as a function of $\mathrm{x}$ ) and similarly for $\Sigma w$ window.

\section{Event-Rate Considerations}

Two important contributers to a digital camera's event-rate characteristics are the rate at which analog pulses can be integrated and digitally-converted and the efficiency with which analog pulse pileup events can be rejected.

Figure 5 illustrates a fast pulse analysis schema which is currently under development. Directly coupled pulses from a photomultiplier unit* undergo conventional delay line clipping (3) to shorten pulse duration and hasten baseline recovery. Fast threshold discriminators and logic circuits provide conventional pulse selections and gates for pulse integrations. Selected photomultiplier are then alternately directed to two (or more) integration sample and hold circuits by means of switches S1 and S2. The buffered photomultiplier charges are similarly multiplexed to an analog-to-digital converter. The net dead time of such a system depends on the number of analog buffers employed and the times required for switching, pulse integration, and digital conversion. With current technology, a dual buffered system could provide about $300 \mathrm{nsec}$ integration time and 10 bit analog-to-digital conversion with a dead time of about $700 \mathrm{nsec}$, for example.

* a unit may comprise a single photomultiplier as in a one-dimensional camera or a row or column of photomultipliers the pulse outputs of which are summed. 




Fig. 5 Fast pulse integration and analog to digital conversion. Modified photomultiplier pulses are alternately directed to two integration sample and hold circuits with $\mathrm{S} 1$ and S2. Buffered photomultiplier charges are similarly multiplexed to analog-todigital converter.

Pulse pileup rejection is usually a function of the analog pulse selection logic, which precedes integration and energy discrimination. Another means of discriminating against pulse pileup, which will be reported in detail elsewhere (4), makes use of the fact that pileup events usually derive from events which are spatially separated. Thus, the light distribution in the scintillator is more dispersed than for a single scintillation event. This manifests in a more distributed partitioning of light among photomultipliers which may be assessed with a number of functions which are sensitive to this distribution change. In the one-dimensional analysis circuitry (Fig. 4) we have experimented with discrimination on the basis of the summation of the photomultipliers' reliability weighting functions, which was electronically readily available. A window based on this function (as a function of $\mathrm{x}$ ) is able to discriminate against pulse pileup events of equal energy which are separated by about one tube diameter. The charac- teristics of this discriminator and others, such as a moment related to the charge distribution, which are more directly related to the partitioning of light are discussed elsewhere (4).

\section{Position Analysis}

To a limited extent two-dimensional position analysis may be treated as two independent one-dimensional position analyses, as is the common practice in analog cameras. However, nonuniform and discontinuous optical and photodetector characteristics give rise to serious coordinate response interdependencies which degrade image position linearity. Figure 6 illustrates a rectangular two-dimensional detector schematically. The photomultipliers are arranged in a cartesian array with the output signals of each column and row summed separate1y. In a first approximation the columnar units are used to compute $x$ in the manner of a one-dimensional detector and similarly for $y$. However, because the photomultiplier unit's $\mathrm{x}$ response curves depend on y and vice versa, an average response function is used to fabricate the table used in this first analyses. A second position analysis calculation employs a larger number of response look up tables which are selected on the basis of the first

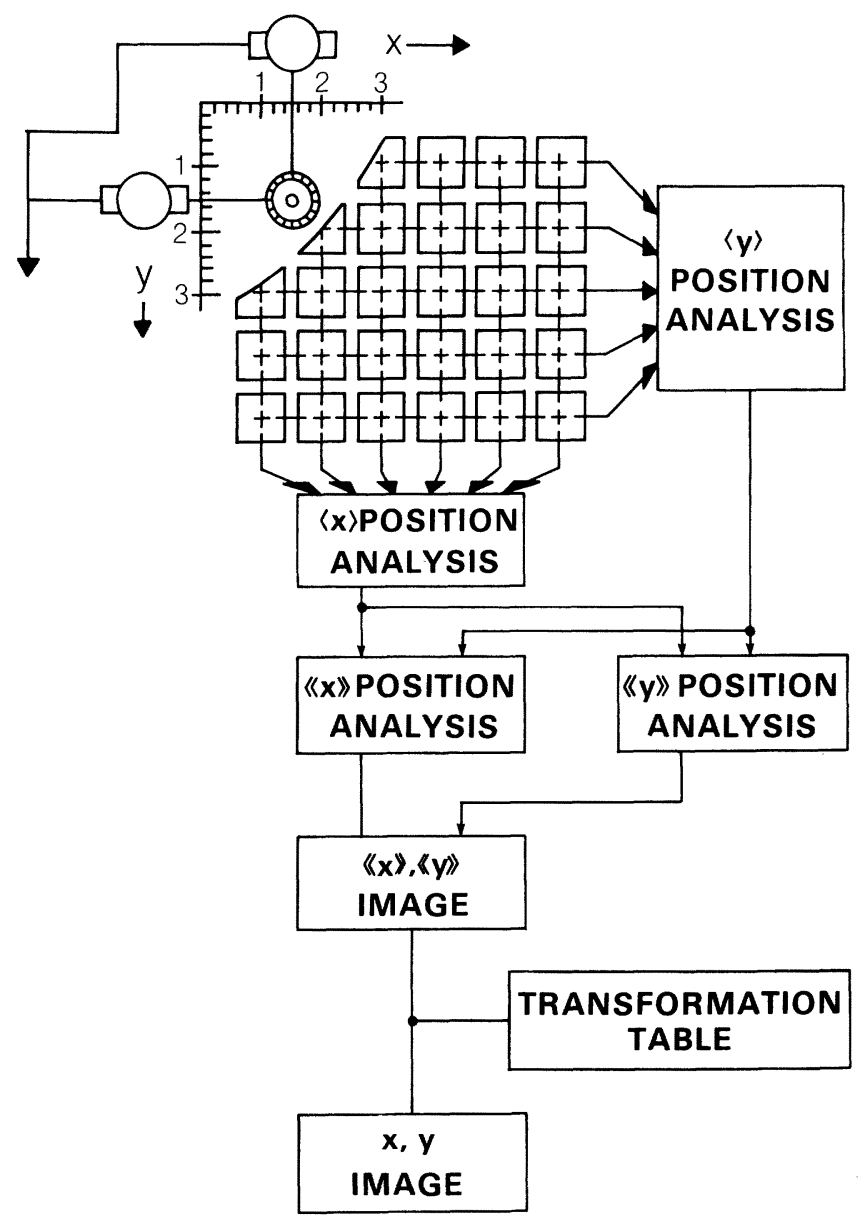

Fig. 6 Rectangular digital camera schematic. Computer positioned narrow-beamed source maps cartesian fieldof-view. First estimated positions, $\langle x\rangle$ and $\langle y\rangle$, are computed in the manner of one-dimensional detectors. Interdependence of coordinate response is accounted for in a second position estimate, $\langle\langle x\rangle\rangle,\langle\langle y\rangle\rangle$. Residual coordinate interdependence is removed by transformation table. 
position estimate $\langle x\rangle,\langle y\rangle$ to account for response interdependence. The degree to which response interdependence is accounted for depends on the number of tables which can be provided. Residual image nonlinearities may be removed by a two-dimensional transformation procedure described elsewhere (1).

The digital circuitry for a two-dimensional iterative position analysis system is outlined in Fig. 7. Normalized pulse charges, $\mathrm{q}^{\mathrm{J}}$ and $\mathrm{q}^{\mathrm{K}}$, from the column and row photomultiplier units are used to compute $\langle\mathrm{x}\rangle$ and $\langle y\rangle$ separately as in Fig. 4. Subsequently, the estimate is compared with the $\mathrm{x}$ coordinate of the centerline of each column of photomultipliers in order to again remove double-valuedness by determining whether $\langle\mathrm{x}\rangle$ is to the left or right $(\mathrm{L} / \mathrm{R})$ of each unit center and similarly for the $\langle y\rangle$ estimate. The importance of this second evaluation of double-valuedness is that it is more statistically reliable than the preliminary procedure in which adjacent photomultiplier's responses are compared (Fig. 4).

At the same time, the $\langle y\rangle$ estimate is used to identify, by number, the response curve (Table Select) which is known (from prior calibration procedures) to most closely describe the response of the photomultiplier columns in the neighborhood of $\langle y\rangle$. The tables are subsequently selected and addressed with $q_{i}{ }^{J}$ and the $L / R$ decisions in order to extract second determinations of $w_{i}{ }^{J}$ and $(w x)_{i}{ }^{J}$. These are used to compute a second estimate of the $x$ position of an event, i.e., $\ll x \gg$, and similarly for $y$.

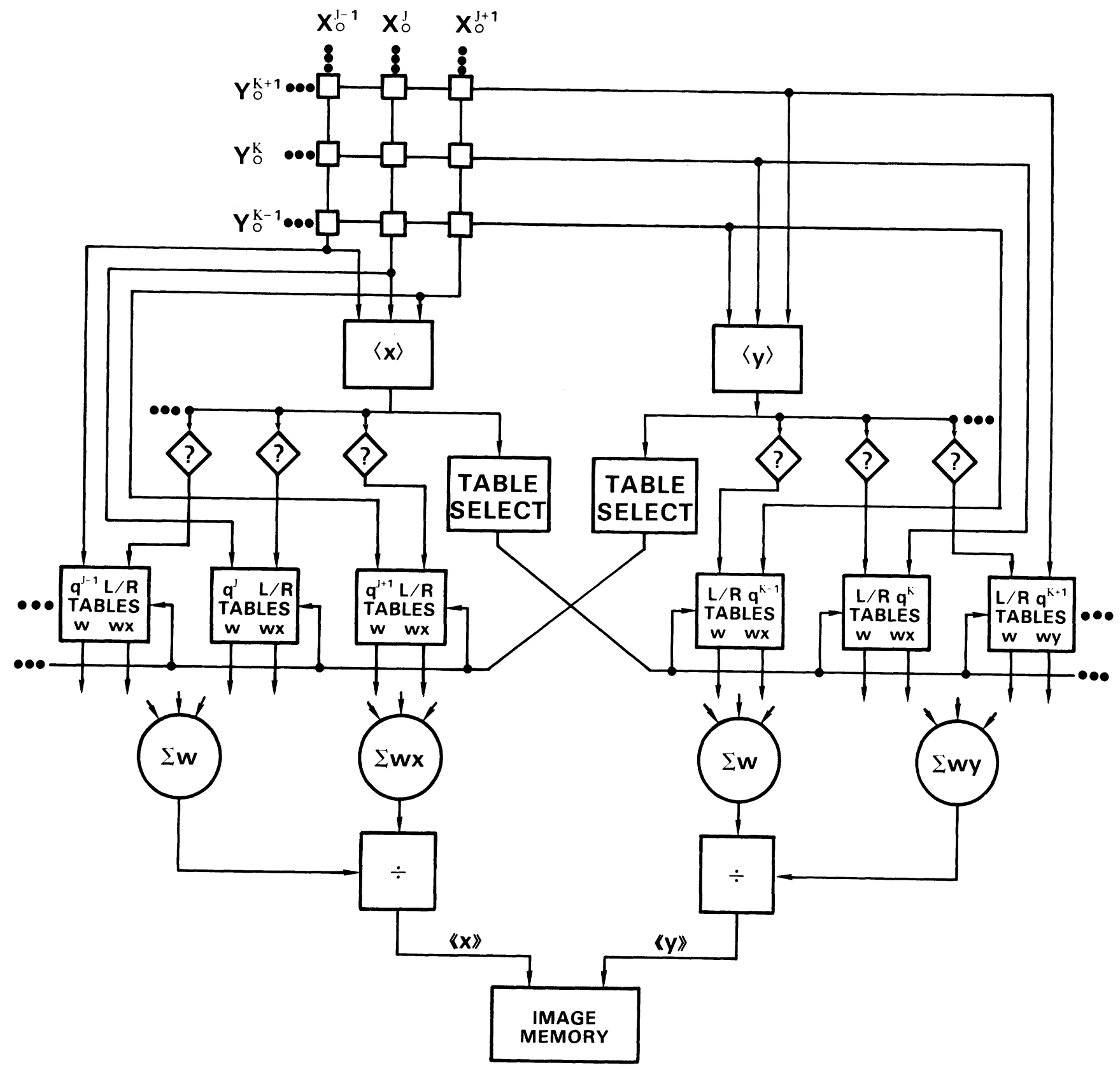

Fig. 7 Two-dimensional digital iterative position analysis. Following a first estimate of positions <x> and <y>, $\langle x\rangle$ is compared, with tube column position $x_{0} J$ to estimate whether event occurred to left (L) or right (R) of center. $\langle y\rangle$ is used to identify response curve which gives best value of $x$ at position $\langle y\rangle$ (prior calibration). Selected table addressed with $\mathrm{q}^{\mathrm{J}}$, etc. to yield coordinate dependent estimate《x》and similarly for $4 y \gg$. 


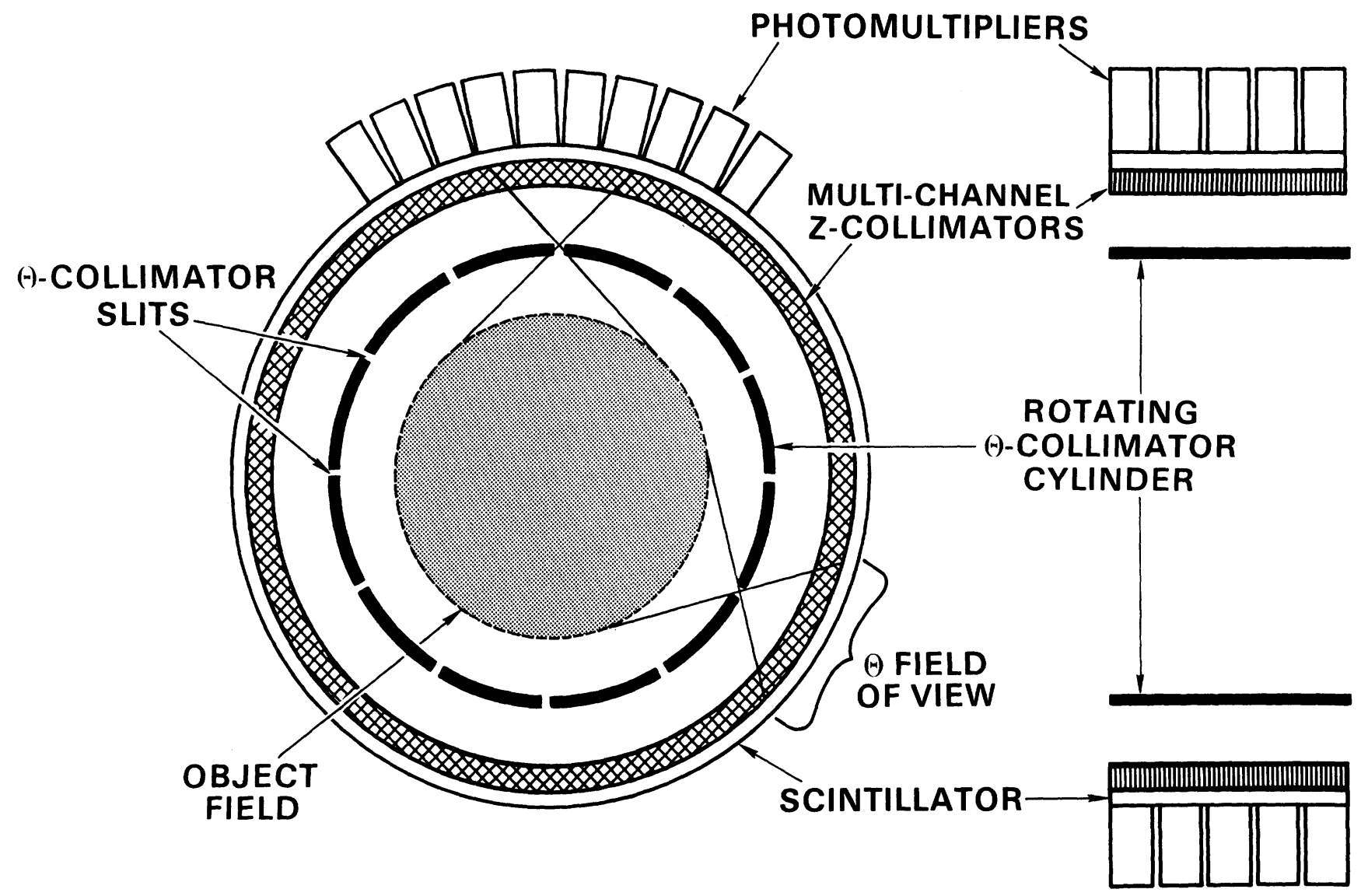

Fig. 8 Dual collimated emission tomography cylindrical camera. A rotatable $\theta$-collimator and stationary z-collimator project nonoverlapping gamma-ray images to inner surface of an unitary cylindrical shell scintillator coupled to parallel rings of photomultipliers. The $\theta$-slit-collimator is incremented through $2 \pi / \mathrm{n}$ radians (where $\mathrm{n}$ equals number of slits) to collect adequate views for reconstruction.

\section{DUAL-COLLIMATED EMISSION TOMOGRAPHY CYLINDRICAL CAMERA}

Figure 8 illustrates schematically the structural design of a stationary cylindrical single crystal scintillation camera which is similar geometrically to the coded aperature multidetector ring imager of Williams, Snapp, and Knoll (5). It comprises a unitary cylindrical shell scintillator which is optically coupled circumferentially to parallel rings of photomultipliers in a $\theta, z$ array. Non-overlapping gamma-ray images are projected to the scintillator's inner surface by a rotatable $\theta$-collimator and a separate stationary $z-$ collimator. The rotatable $\theta$-collimators consist of a family of equidistant slits cut parallel to the $z$ axis on a rotatable cylindrical lead she1l. The multiple slit shell is positioned between the scintillator and the cylindrical field-of-view. Its diameter is chosen to provide a number of nonoverlapping $\theta$-views, magnification, and field-of-view desired. A stationary multichannel planar z-collimator is contiguous to the scintillator's inner surface.

The number of nonoverlapping $\theta, z$ views imaged at fixed $\theta$-collimator position is equal to the number of slits (12 shown). Thus, a complete set of single photon tomographic reconstruction data is obtainable, by rotation through $2 \pi / \mathrm{n}$ radians, where $\mathrm{n}$ equals the number of slits. The design permits fast $2 \pi$ imaging without distortion due to comera motion.

Position analysis by digital means is essentially equivalent to that of a rectangular camera and should yield comparable inherent resolution and uniformity characteristics. Higher event-rate can be achieved, however, because only a relatively small number of photomultipliers share light from a scintillation and thus only a fraction of the amera need be made dead as a result of a scintillation event. On this basis and with fast charge intergration-digital conversion and digital pulse pileup rejection procedures, in vivo event-rates of about 600,000 events/sec with minimal pileup distortion should be possible. Such a characteristic could make multiple slice cardiac blood pool imaging a practical reality.

\section{ACKNOWLEDGEMENT}

This work was supported by the Veterans Administration Research Service.

\section{REFERENCES}

1. Genna S, Pang SC, Smith A: Digital scintigraphy: principles, design and performance. J Nucl Med 22: 365-371, 1981.

2. Muehllehner G: The impact of digital technology on the scintillation camera. J Nuc1 Med 22:389-391, 1981.

3. Blatt SL, Mahieux J, Kohler D: Elimination of pulse pileup distortion in nuclear radiation spectra. Nuc1 Inst Meth 60:221-230, 1968.

4. Pang SC, Genna S: In preparation.

5. William JJ, Snap WP, Knoll GF: Introducing sprint: A single photon ring system for emission tomography. IEEE Trans Nucl Sci NS-26, No. 1, 1979. 